\title{
Chromosome 18 pairing behavior in human trisomic oocytes. Presence of an extra chromosome extends bouquet stage
}

\author{
I Roig ${ }^{1}$, P Robles ${ }^{1}$, R Garcia ${ }^{1}$, I Martínez-Flores ${ }^{1}$, LI Cabero ${ }^{2}$, J Egozcue ${ }^{1}$, B Liebe ${ }^{3}$, \\ $\mathrm{H}_{\text {Scherthan }}{ }^{3,4}$ and M Garcia ${ }^{1}$ \\ ${ }^{1}$ Departament de Biologia Cellular, Fisiologia i Immunologia, Universitat Autònoma de Barcelona, \\ 08193-Bellaterra, Spain, ${ }^{2}$ Servei de Ginecologia i Obstetrícia, Hospital Materno-infantil de la Vall d'Hebron, \\ Barcelona, Spain, ${ }^{3}$ Max-Planck-Institute for Molecular Genetics, Ihnestrasse 73, D-14195 Berlin, Germany and \\ ${ }^{4}$ Institute of Radiation Biology Bw Neuherbergstr. 1180937, München, Germany \\ Correspondence should be addressed to M Garcia, Unitat de Biologia, Facultat de Medicina, Universitat Autònoma de \\ Barcelona,08193-Bellaterra, Spain; Email: montserrat.garcia.caldes@uab.es
}

\begin{abstract}
Little is known about the first meiotic prophase stages in the human female because these occur during fetal life, and only a few studies have addressed aneuploid human oocytes. In this paper, the synaptic process in the meiotic prophase in three 47, $X X+18$ cases is analyzed. A complete study of the dynamics of centromeres and telomeres, cohesin core and synapsis development in aneuploid female meiosis was performed. Investigation of chromosome dynamics in prophase of trisomy 18 oocytes show that these events follow the major patterns seen earlier in euploid oocytes. However, there is a significant delay in the resolution of bouquet topology which could relate to the presence of a surplus chromosome 18 axial element in zygotene oocytes. Pachytene oocytes displayed normal synapsis among the three chromosome 18s. However, in some oocytes the surplus chromosome 18 core was aligned to the bivalent 18. As ataxia telangiectasia and Rad3 related kinase (ATR) has been described as a marker for late-pairing chromosomes in mice, ATR distribution was analyzed in human meiocytes spermatocytes, euploid oocytes and trisomic oocytes. In contrast to the observations made in mice, no preferential staining for late-pairing chromosomes was observed in humans. In the cases studied, bivalent synapses progressed as in a normal ovary, contrasting with the hypothesis that a surplus chromosome can modify pairing of other chromosomes.

Reproduction (2005) 129 565-575
\end{abstract}

\section{Introduction}

Meiosis is the special cell division by which a chromosome complement is reduced to the haploid number in order to preserve diploidy at fertilization. During first meiotic prophase, homologous chromosomes pair and exchange material between them. Paired homologs develop a tripartite structure between them, the synaptonemal complex (SC) (Fawcett 1956, Moses 1968, Schmekel \& Daneholt 1995). The SC is formed by SYCP proteins (Synaptonemal Complex Proteins, SYCP/SCP; Heyting 1996). SYCPs are laid down on a cohesin scaffold which is established during the pre-meiotic S-phase (Eijpe et al. 2000, 2003, Pezzi et al. 2000, Pelttari et al. 2001, Prieto et al. 2001, 2002, 2004).

At the leptotene stage, SYCP3 and SYCP2 form an axial element (AE) along each chromosome (Lammers et al. 1994, Offenberg et al. 1998). During this stage, initiation of recombination can be monitored by immunostaining of the phosphorylated version of histone $\mathrm{H} 2 \mathrm{AX}(\gamma \mathrm{H} 2 \mathrm{AX})$ (Mahadevaiah et al. 2001, Roig et al. 2004, Lenzi et al. 2005). At the zygotene stage, homolog pairing starts and SYCP1 organizes the central element of the SC by developing transverse filaments between closely aligned regions of AEs (Meuwissen et al. 1992); from this time on, AEs are called lateral elements (LEs). In order to promote homologous synapsis, chromosome ends (telomeres) cluster at a limited portion of the nuclear envelope, known as bouquet topology (for reviews see Scherthan 2001 and Harper et al. 2004). Studies in cattle and human have revealed that bouquet topology lasts longer in the mammalian female than in the male (Bojko 1983, Pfeifer et al. 2003, Roig et al. 2004). At pachytene, all homologs are completely paired (bivalents). Finally, at diplotene, SCs almost completely dissolve. The substages of prophase I are characterized by the initiation and progression of recombination which can be followed by the dynamic turnover 
of repair proteins (reviewed in Cohen \& Pollard 2001, Moens et al. 2001).

In mammalian males meiosis is a continuous process, while in the female meiotic prophase occurs during early fetal life, and arrests before birth at a specialized diplotene-dictyotene stage. The meiotic division is blocked until the female enters puberty. Despite the difficulties associated with the study of human female meiotic prophase, some aneuploid ovaries have been studied using classical staining techniques (Luciani et al. 1976, Wallace \& Hulten 1983, Speed 1984), fluorescent in situ hybridization (FISH) (Cheng et al. 1995, 1998) or immunofluorescent (IF) (Barlow et al. 2002) techniques.

In these studies, pairing anomalies implying the presence of the extra chromosome are described. Nevertheless, in all cases, at least two homologs seem to pair normally forming a bivalent, and even pairing of the three homologs (trivalent formation) has been described in all studied cases, with different efficiency (Luciani et al. 1976, Wallace \& Hulten 1983, Speed 1984, Cheng et al. 1995, 1998, Barlow et al. 2002). In some of these papers a disturbing effect of the extra chromosome on the pairing process of other homologs is suggested (Cheng et al. 1998).

In those studies in which the pairing process is analyzed in silver-stained preparations (Wallace \& Hulten 1983, Speed 1984), a thickening of the unpaired axial element (univalent) of the extra chromosome is seen, suggesting a different proteinaceous composition. This phenomenon is not restricted to humans, and has also been observed in rat meiocytes (Martínez-Flores et al. 2001). However, recent studies in which IF techniques have been applied to stain SYCP3 in human trisomic 21 oocytes have not observed any univalent thickening, suggesting that SYCP3 is not implicated in this phenomenon (Barlow et al. 2002).

Trisomy 18 syndrome, also known as Edwards' syndrome, has a meiotic maternal origin in $89 \%$ of cases (Hassold \& Hunt 2001) and causes major physical abnormalities, growth delay and severe mental retardation. Very few affected children survive beyond their first year.

The aim of the present study was to analyze the pairing process of homologs in ovaries from three trisomic
18 fetuses in order to assess the effect of the presence of an extra chromosome 18 on the progress of chromosomal events in the female meiotic prophase I. To this end, immunofluorescent staining (IF) to SYCP1 and SYCP3 proteins, chromosome painting, and centromere and telomere FISH were performed. As it has been recently described that human SCs are also formed of a cohesin axis (see above), analysis of a specific meiotic cohesin REC8 (Prieto et al. 2004) was performed in order to check whether cohesins are responsible for the unpaired univalent thickening observed in previous studies (Wallace \& Hulten 1983, Speed 1984, Martínez-Flores et al. 2001).

Furthermore, as ataxia telangiectasia and Rad3 related kinase (ATR) has been described as a marker for late-pairing chromosomes in mouse pachytene spermatocytes (Moens et al. 1999, Baart et al. 2000), IF to ATR was performed to investigate its distribution in aneuploid oocytes. Due to the absence of any published data about ATR in humans, ATR distribution in human euploid spermatocytes and oocytes is also described.

\section{Materials and Methods \\ Tissue origin and processing}

Three fetal cases prenatally diagnosed as Edwards' syndrome (V62, V63 and V70) and six euploid cases (V54, V93, V94, V98, V106 and V107) (Table 1) were used after legal interruption of pregnancy according to the Ethics Rules Committee of the Hospital de la Vall d'Hebron of Barcelona, Spain. The age of each case was calculated from the last menstrual period and from echography.

Ovaries were processed as described by Roig et al. (2003) in order to obtain a somatic karyotype of the sample. Paraffin embedding, tissue sections, structurally preserved nuclei preparations and oocyte spreads for IF and FISH purposes were performed as recently described (Martínez-Flores et al. 2003, Roig et al. 2004). Human testis from an adult male with proven fertility was processed as previously described (Scherthan et al. 1996).

Table 1 Biological material, karyotype and bouquet frequencies.

\begin{tabular}{|c|c|c|c|c|}
\hline Case & Gestational weeks & Diagnosis & Karyotype & Oocytes at bouquet stage \\
\hline V54 & 19 & Cardiopathology & $46, X X^{a}$ & NA \\
\hline V93 & 19 & Osteochondrodysplasia & $46, X^{b}$ & $36.48 \%(n=74)$ \\
\hline V94 & 20 & Neural tube defect & $46, X^{b}$ & $28.00 \%(n=75)$ \\
\hline V98 & 19 & Muscular dystrophy & $46, X X^{b}$ & $34.21 \%(n=76)$ \\
\hline V106 & 21 & Encephalocoele & $46, X X^{b}$ & $35.61 \%(n=73)$ \\
\hline V107 & 19 & Cardiopathology & $46, X^{b}$ & $37.66 \%(n=77)$ \\
\hline V62 & 21 & Edwards' syndrome & $47, X X+18^{a}$ & $47.06 \%(n=102)$ \\
\hline V63 & 20 & Edwards' syndrome & $47, X X+18^{a}$ & $56.00 \%(n=75)$ \\
\hline V70 & 19 & Edwards' syndrome & $47, X X+18^{a}$ & $54.67 \%(n=75)$ \\
\hline
\end{tabular}

${ }^{a}$ Karyotype obtained from ovarian stromal somatic culture. ${ }^{b}$ karyotype obtained from prenatal diagnosis. NA, not analyzed. 


\section{DNA probes and labeling}

Centromeres of all human chromosomes were detected by FISH with alpha satellite-DNA sequences (pan-centromeric probe), as obtained by alphoid-specific PCR from genomic human DNA (Weier et al. 1991) and were labeled with Cy3-dUTP by random priming. All chromosome telomeres were detected after applying FISH with an Fluorescien (FITC)-conjugated $\left(\mathrm{CCCTAA}_{3}\right)$ peptide nucleic acid (PNA) telomere probe (DAKO, Glostrup, Denmark; pan-telomeric probe).

Commercial Whole Chromosome 18 Probe (WCP) Cy3-conjugated and Whole Chromosome 13 Probe Cy3conjugated (Cambio, Cambridge, UK), as well as a dual locus-specific identification (LSI) probe 13/21 (Oncor, Qbiogene, Irvine, CA, USA) and a locus-specific biotinylated probe for the $18 \mathrm{q}$ subtelomeric region (YAC 932Q10) were used to analyze the homolog-pairing process.

DNA was counterstained applying an antifade solution (Vector Laboratories) containing $0.1 \mu \mathrm{g} / \mathrm{ml}$ DAPI $\left(4^{\prime}, 6^{\prime}\right.$-diamidino-2-phenylindole; Sigma).

\section{Fluorescent in situ hybridization (FISH) on methanol:acetic acid spread oocytes}

In order to study the homolog chromosome 18 pairing process, FISH was performed on methanol:acetic acid spread oocytes as described before with slight modifications (Barlow \& Hultén 1997). Slides were post-fixed in a formaldehyde solution and denatured in $70 \%$ formamide in $2 \times$ SSC at $69^{\circ} \mathrm{C}$ for $4 \mathrm{~min}$. Probe denaturation was performed as described by the manufacturer and the denatured probe was applied after slide dehydration. Three post-hybridization washes were performed in $50 \%$ formamide in $2 \times$ SSC, $2 \times$ SSC and $0.4 \times$ SSC $0.05 \%$ Tween 20 at $45^{\circ} \mathrm{C}$. LSI 18 probe detection was performed applying Avidin FITC-conjugated antibody (1/150) for $1 \mathrm{~h}$ at $37^{\circ} \mathrm{C}$.

In this part of the study 719 oocytes were analyzed. Oocyte staging was performed according to the morphological criteria previously described (Garcia et al. 1987).

\section{Immunofluorescent (IF) staining}

The axial elements of the SCs were identified with a rabbit polyclonal serum against SYCP3 (Meuwissen et al. 1992). Homolog synapsis was tracked by marking the central element of the SC with a rabbit polyclonal serum against SYCP1 (Lammers et al. 1994) (both were kind gifts of Christa Heyting, Wageningen, The Netherlands). The cohesin axis was detected using a mouse polyclonal serum against cohesin REC8 (Prieto et al. 2004, a kind gift of José Luís Barbero, DIO/CNB, Trescantos, Madrid, Spain). Staining of all chromosome telomeres was performed applying a mouse monoclonal antibody (Imgenex, San Diego, CA, USA) against telomeric protein TIN2 (Kim et al. 1999). Finally, ATR dynamics were described using a rabbit polyclonal antibody against ATR (Abcam, Cambridge, UK).
IF staining was performed as described by Roig et al. (2004). Primary antibodies were diluted in PTBG (PBS, $0.2 \%$ BSA, $0.2 \%$ gelatin, $0.05 \%$ Tween 20 ) and incubated overnight at $4{ }^{\circ} \mathrm{C}$ in a humid chamber.

After washing away unattached antibodies with PBTG, detection was performed using some of the following fluorochrome-conjugated secondary antibodies (diluted in PTBG; all Jackson ImmunoResearch Laboratories, West Grove, PA, USA): goat anti-rabbit Cy3, goat anti-rabbit FITC antibody, goat anti-rabbit Cy5, a goat anti-mouse Cy3 antibody, a goat anti-mouse FITC antibody and a goat antimouse Cy 5 antibody. Secondary antibodies were incubated for one hour at $37^{\circ} \mathrm{C}$ in a humid chamber. Later, excess secondary antibodies were washed off with PBTG and the fluorescent signals were fixated with $1 \%$ formaldehyde in PBS. DNA was counterstained as mentioned above.

\section{FISH on immunostained preparations}

IF stained preparations were hybridized with an $18 q$ subtelomeric probe as described by Scherthan et al. (1996) with modifications. DAPI staining was removed by washing for $5 \mathrm{~min}$ in water. A first denaturation of the slide was performed in $70 \%$ formamide in a $2 \times$ SSC solution for $5 \mathrm{~min}$ at $70^{\circ} \mathrm{C}$. The slide was then washed in water and a 3-h NaSCN (Sodium Thiocyanate) $1 \mathrm{M}$ treatment was applied at $65^{\circ} \mathrm{C}$. A second denaturation was then performed as mentioned before. Dehydration of the slide was carried out before applying the denatured probe to the slide. Hybridization was performed at $37^{\circ} \mathrm{C}$ in a humid chamber for at least $48 \mathrm{~h}$. Slide washes and probe detection were performed as described above.

\section{Microscopy and image analysis}

Preparations were evaluated using an Olympus BX70 fluorescence microscope (Olympus Optical Co.). Images were captured and produced by Smart Capture software. (Vysis, Grove, IL, USA) Images were further processed using Adobe Photoshop (Adobe, San Jose, USA) to match the fluorescent intensity seen in the microscope.

\section{Results}

\section{Sample karyotypes obtained from stromal ovarian culture agree with prenatal diagnosis}

For the cases V54, V62, V63 and V70, a stromal ovarian culture was established as described before (Roig et al. 2003 ) in order to certify prenatal diagnosis and the karyotype of the respective case. In all of the Edwards' syndrome cases, ovarian cultures revealed a $47, X X+18$ karyotype (Table 1). The karyotype of V54 obtained from the somatic culture was $46, \mathrm{XX}$, thus validating this sample as a control case (Table 1).

\section{Chromosomal dynamics in trisomy 18 oocytes}

IF staining for SYCPs, telomeric proteins, as well as a dualcolor FISH applying pan-centromeric and pan-telomeric 
probes performed on structurally preserved oocyte preparations and ovary sections respectively, were carried out in order to check for pairing progression in trisomy 18 oocytes. These experiments revealed the same chromosome and protein dynamics as described in euploid oocytes (Fig. 1) (Roig et al. 2004).

SYCP3 first appeared at the preleptotene stage as aggregates in DAPI-negative regions, most likely nucleoli. At this stage, centromeres were located at the periphery of the nucleus, while telomeres where found inside the nucleus. At the leptotene stage, telomeric signals were located at the nuclear periphery, while SYCP3 started forming curly threads. At the leptotene/zygotene transition, telomeric clustering was first observed (Fig. 1A). However, tight telomeric clustering was not found until the zygotene stage (Fig. 1B). At this stage, synapsis was initiated and the first SYCP1 fibers were seen mainly near the bouquet base, although some interstitial pairing was also seen (Fig. 1C). At the pachytene stage nuclear polarization was resolved and complete pairing existed and SYCP1 was found along the entire length of the synaptonemal complex. At the diplotene/dictyotene stage, SYCP1 was found as little dots and SYCP3 as small threads, telomeres were found inside the nuclei and centromeres were sometimes seen in a line-like organization, as was reported in the euploid human oocytes (Roig et al. 2004).

\section{Bouquet topology is increased in trisomic oocytes}

In order to compare meiotic prophase progression in aneuploid and euploid cases, the percentage of oocytes displaying a bouquet topology in each case was analyzed. In the trisomic 18 cases, $47 \%, 56 \%$ and $55 \%$ of the oocytes (Table 1) had their telomeres clustered in a limited sector of the nuclear periphery (bouquet topology; Fig. 1). In euploid cases of a similar developmental stage, the bouquet proportion was 36\%, 28\%, 34\%, 36\% and 38\% (Table 1). Thus, a statistically significant $(P<0.0001)$ increase in the proportion of the bouquet stage oocytes per ovary in trisomy 18 cases was observed.

As noted before in euploid oocytes (Table 1 and Roig et al. 2004), the trisomy 18 oocyte bouquet frequencies
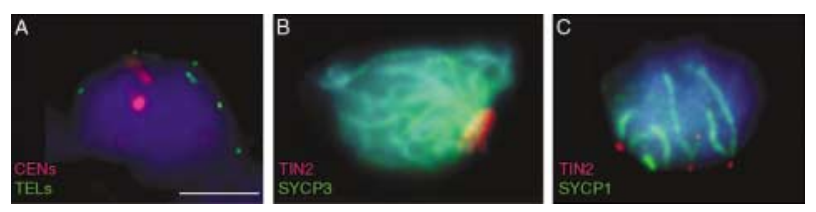

Figure $13 \mathrm{D}$ structure-preserved $(\mathrm{A}-\mathrm{C})$ trisomic oocytes displaying all centromeres and all telomeres obtained by FISH (A) and SYCPs and all telomeres (TIN2) revealed by IF (B-C). (A) Equatorial plane of a leptotene-zygotene transition oocyte FISHed with pan-centromeric (red) and pan-telomeric (green) probes showing initiation of telomeric clustering. In this focal plane just some telomere and centromere signals are present. (B) Focal plane of a zygotene oocyte displaying a tight telomeric clustering, that is, the bouquet. (C) Zygotene oocyte showing initiation of synapsis as visualized by the appearance of the first SYCP1 threads (green). Bar, $10 \mu \mathrm{m}$. were statistically significantly increased $(P<0.0001)$ over those observed in human spermatogenesis.

\section{Cohesin core and SC organization during aneuploid meiotic prophase}

Silver-stained preparations of trisomic oocytes revealed a significant thickening of the unpaired univalent (Wallace \& Hulten 1983, Speed 1984). Thus, REC8 distribution throughout the meiotic prophase in trisomic 18 oocytes was analyzed to see whether this phenomenon was caused by cohesin proteins and whether aneuploidy could affect organization of cohesin cores. In order to properly follow homolog synapsis, IF staining was also performed on the major component of the central element of the SC (SYCP1).

REC8 first appeared at the preleptotene stage as a diffuse signal throughout the nucleus (not shown). At the leptotene stage, REC8 was organized as thin, curly threads (Fig. 2A). At the zygotene stage, REC8 was present as thin threads in unsynapsed regions and as thicker threads which colocalize with SYCP1 corresponding to synapsed regions between homologs (Fig. 2B). At the pachytene stage, complete colocalization of REC8 and SYCP1 was seen in the majority of the oocytes (Fig. 2C, see below). At the diplotene stage, SYCP1 started to disappear and
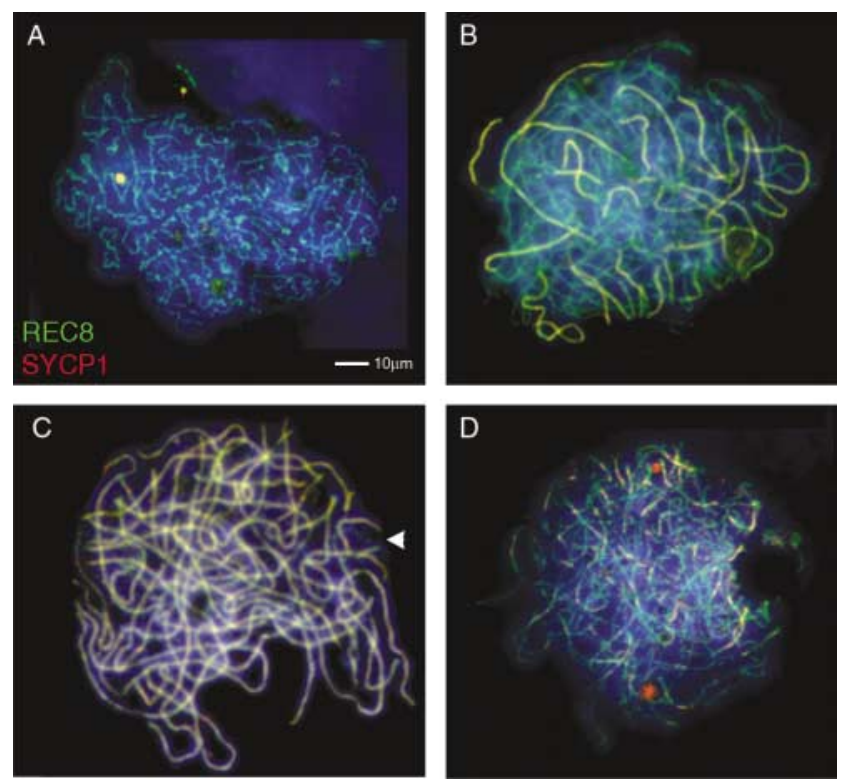

Figure 2 Dual color IF against REC8 (green) and SYCP1 (red) was performed on trisomic 18 oocytes in order to analyze cohesin REC8 distribution along the meiotic prophase. (A) Leptotene oocytes displaying long, thin, curly REC8 threads. (B) Zygotene oocyte with REC8 and SYCP1 colocalization at synapsed regions of the homologous chromosomes. (C) Pachytene oocyte in which complete colocalization of SYCP1 and REC8 was found along the synapsed chromosomes; note that this oocyte presents an unsynapsed $\mathrm{AE}$ (arrowhead), most likely a chromosome 18. (D) Diplotene oocyte presenting unsynapsed regions and still some synapsed regions, where colocalization between SYCP1 and REC8 persists; note that two small SYCP1 aggregates are present. 
REC8 persisted as long, thin threads in SYCP1-devoid regions (Fig. 2D).

When analysis of REC8 and SYCP1 distribution in trisomic 18 oocytes was performed, some pachytene-stage oocytes displaying complete synapsis of the homologs while the three chromosome 18 cores were partially aligned with synapsed and asynapsed regions, were found (Fig. 3).

\section{Pairing and synapsis of the three chromosome 18s during the meiotic prophase}

In order to analyze the pairing process of the chromosome $18 \mathrm{~s}$ in more detail, dual-color FISH using a WCP 18 and a subtelomeric $18 q$ probe was performed on methanol:acetic acid oocyte preparations (Fig. 4, Table 2). In total, 193 oocytes were analyzed.

At the leptotene stage (Fig. 4B), most oocytes (71.43\%) displayed three independent signals for the paint and the subtelomeric region. The remaining oocytes displayed two signals, one corresponding to two joint subtelomeric
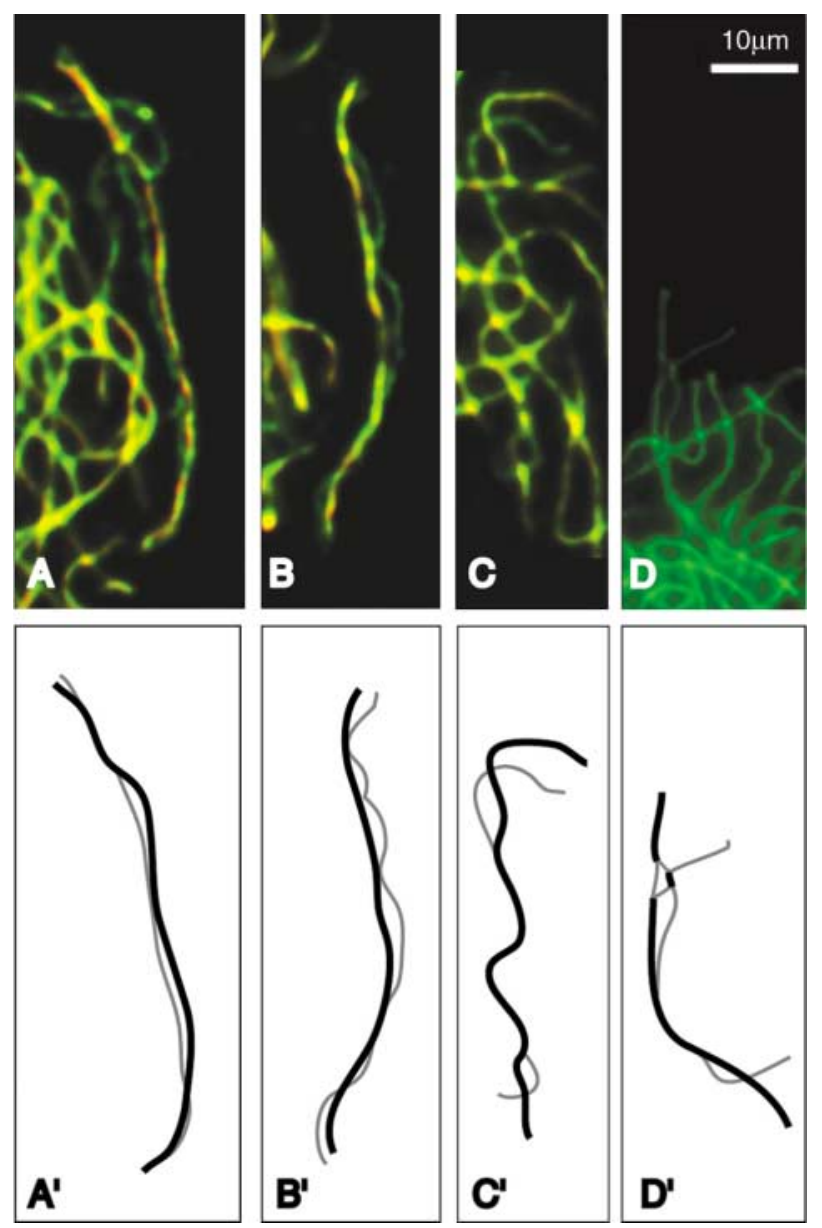

Figure 3 Different associations between the homolog $18 \mathrm{~s}$ observed in pachytene-stage oocytes. IF against REC8 (green) and SYCP1 (red) (A, B and C) and SYCP3 (green) (D) is shown. A schematic drawing is also shown $\left(A^{1}, B^{1}, C^{1}\right.$ and $\left.D^{1}\right)$; black, paired regions; gray, unpaired regions. Image $\mathrm{D}$ is a fusion of two focal planes.
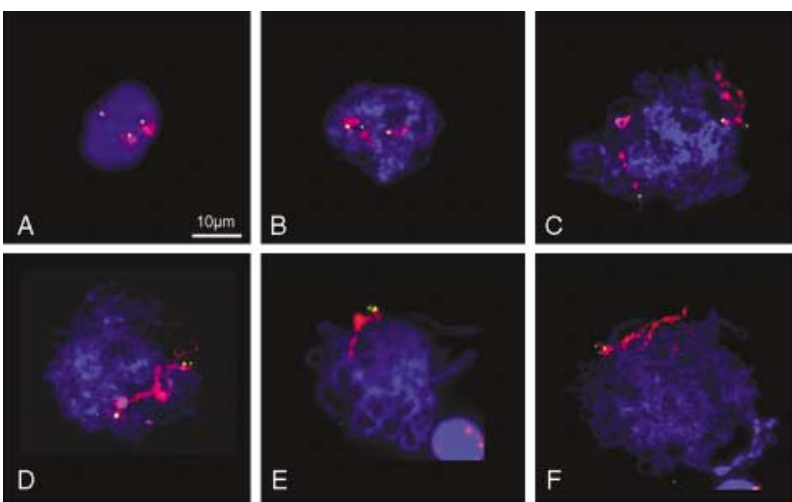

Figure 4 Pairing process of the homolog 18 s analyzed by applying FISH with WCP 18 (red) and LSI 18 (green) on methanol:acetic acid spread oocytes. (A) Interphase nucleus. (B) Early leptotene stage with three independent signals. (C) Zygotene-stage oocyte with two homo$\log 18$ s pairing and the other one separate. (D) Zygotene-stage oocyte in which the trivalent 18 is forming; note that one chromosome 18 (left) is pairing with an existing bivalent (right). (E) Pachytene oocyte showing a trivalent 18. (F) Diplotene oocyte where the three chromosome $18 \mathrm{~s}$ remain joined, presumably by the chiasmata.

signals colocalizing with one paint 18 signal (consistent with an already paired bivalent 18) and the other was the unpaired third chromosome 18 (univalent 18). During the zygotene stage (Fig. 4C,D), 22\% of oocytes had three signals (unpaired chromosome 18). Two signals (bivalent plus univalent) were seen in $37 \%$ of oocytes. Finally, one single signal comprising all three chromosome 18 paired (trivalent) was seen in $42 \%$ of oocytes. At the pachytene stage, the vast majority of the oocytes analyzed (92\%) displayed a trivalent (Fig. 4E). The remaining $8 \%$ of oocytes showed two separate signals corresponding to a bivalent plus a univalent. Diplotene-stage oocytes generally displayed only one signal (83.33\%) (Fig. 4F), but oocytes with two separate chromosome 18 signals were also observed.

The high frequency of single signals observed at the pachytene stage contrasted with previous studies performed on trisomic 21 oocytes (Luciani et al. 1976, Wallace \& Hulten 1983, Speed 1984, Barlow et al. 2002). Single signals observed in methanol:acetic acid preparations at the pachytene stage could either come from synapsed chromosomes or from aligned homologs. Thus, to determine if pairing or alignment of the extra chromosome existed, FISH, using a subtelomeric $18 q$ probe on REC8 and SYCP1 IF preparations, was performed (Fig. 5). All the pachytene oocytes analyzed $(n=10)$

Table 2 Distribution of chromosome 18 signals in methanol:acetic acid spread oocytes from case V70.

\begin{tabular}{lccc}
\hline Stage & $\begin{array}{c}\text { Oocytes with } \\
\text { a trivalent } \\
(n(\%))\end{array}$ & $\begin{array}{c}\text { Oocytes with a } \\
\text { bivalent and a } \\
\text { univalent }(n(\%)))\end{array}$ & $\begin{array}{c}\text { Oocytes with } \\
\mathbf{3} \text { univalents } \\
(n(\%)))\end{array}$ \\
\hline Leptotene & $0(0.00)$ & $8(28.57)$ & $20(71.43)$ \\
Zygotene & $22(41.67))$ & $25(36.67)$ & $13(21.67)$ \\
Pachytene & $91(91.92)$ & $8(8.08)$ & $0(0.00)$ \\
Diplotene & $5(88.33)$ & $1(16.67)$ & $0(0.00)$ \\
\hline
\end{tabular}



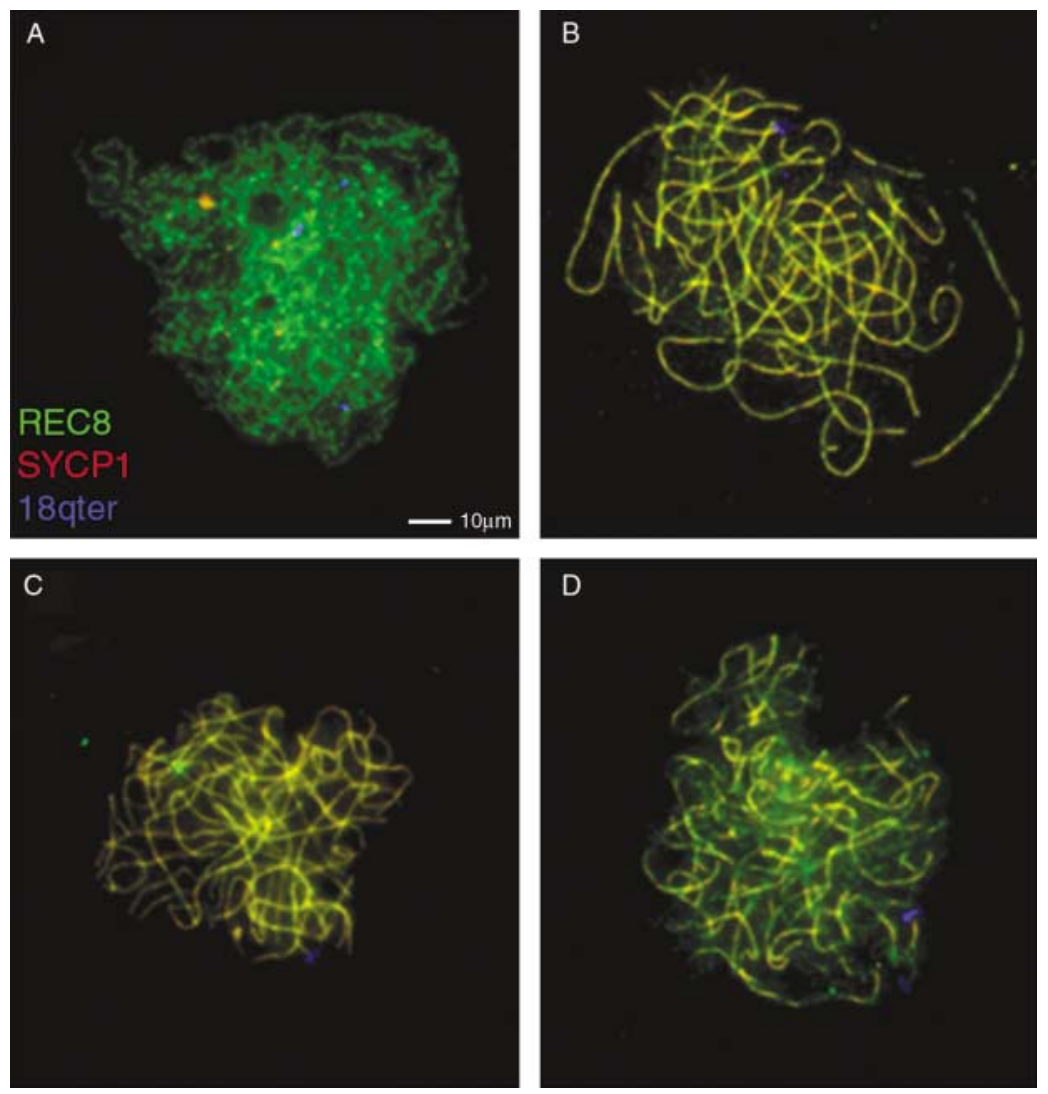

Figure 5 Human oocytes stained for REC8 (green) and SYCP1 (red) were FISHed with an 18q subtelomeric probe (pseudocolored blue). (A) Leptotene oocyte displaying three univalent 18s. (B) Late-zygotene oocyte having a bivalent (seen in yellow due to the colocalization of the SYCP1 and REC8) plus a univalent 18. (C) Pachytene oocyte with a trivalent 18. (D) Diplotene oocyte with two separate signals for the subtelomeric $18 \mathrm{q}$ region. Note that there are still portions where the three chromosome $18 \mathrm{~s}$ are still paired. showed a single $18 q$ subtelomeric signal matching with a SYCP1 thread (Fig. 5C). Despite the small number of nuclei studied, this value is almost the same as that observed by $\mathrm{FISH}$ in the previous experiment on methanol:acetic acid spread preparations (Table 2). Similarly, all the leptotene oocytes $(n=5)$ presented three unsynapsed chromosome $18 \mathrm{~s}$ (Fig. 5A). Fifty percent of the zygotene oocytes analyzed $(n=40)$ displayed a bivalent 18 plus a univalent 18 (Fig. 5B). Only two diplotene oocytes could be analyzed one showed a bivalent plus a univalent configuration, the other presented a desynapsed trivalent (Fig. 5D).

\section{Does the presence of an extra chromosome affect the pairing process of other homologs?}

In order to analyze the effect of the presence of three chromosome $18 \mathrm{~s}$ in the nucleus and to see if this phenomenon affects the pairing of other homolog pairs, FISH was performed using a WCP 13 plus an LSI 13/21 to follow the chromosome 13 pairing process in trisomy 18 oocytes (V70). The results (Table 3) showed that chromosome $13 \mathrm{~s}$ were not paired at leptotene $-100 \%$ of the studied oocytes had two univalents. Chromosome 13s paired during the zygotene stage, where we observed $70 \%$ of the oocytes displaying one bivalent signal. At the pachytene stage, all nuclei analyzed displayed one chromosome 13 bivalent.

The same experiment was performed in a euploid case from a similar developmental stage (V54) (Table 1).
The results showed no significant differences to those obtained in trisomic 18 oocytes. Almost all leptotene oocytes analyzed displayed two chromosome 13 univalents $(91 \%)$. Seventy percent of the zygotene oocytes showed one chromosome 13 bivalent. All pachytene and diplotene oocytes showed only one chromosome 13 bivalent.

\section{ATR location in human meiosis}

It has been proposed that ATR controls the correct pairing of the homologs in the male mouse, as has been found preferentially in late-pairing chromosomes in pachytenestage spermatocytes (Keegan et al. 1996, Moens et al. 1999). Therefore, and given the delayed formation of the trivalent 18 (Fig. 3), the ATR dynamics in trisomic 18 oocytes were studied.

\section{ATR distribution in euploid human spermatocytes}

As ATR detection has only been performed on mouse spermatocytes, ATR distribution in human spermatocytes was first analyzed in order to characterize the location of this protein during male meiotic prophase (Fig. 6). Spermatocyte staging was performed according to parameters described previously (Solari 1980).

ATR was first seen in human spermatocytes at the leptotene stage as a few foci on the AEs. At zygotene, the number of ATR foci substantially increased and were located in unsynapsed and synapsed regions up to early 
Table 3 Distribution of chromosome 13 signals in methanol:acetic acid spread oocytes from cases V70 and V54.

\begin{tabular}{|c|c|c|c|c|c|c|}
\hline \multirow[b]{2}{*}{ Stage } & \multicolumn{3}{|c|}{ V70 } & \multicolumn{3}{|c|}{ V54 } \\
\hline & $\begin{array}{c}\text { Sub-stage } \\
\text { proportion (\%) }\end{array}$ & $\begin{array}{c}\text { Oocytes with } \\
\text { a bivalent }(n(\%))\end{array}$ & $\begin{array}{c}\text { Oocytes with } \\
\text { two univalents }(n(\%))\end{array}$ & $\begin{array}{c}\text { Sub-stage } \\
\text { proportion (\%) }\end{array}$ & $\begin{array}{c}\text { Oocytes with } \\
\text { a bivalent }(n(\%))\end{array}$ & $\begin{array}{l}\text { Oocytes with two } \\
\text { univalents }(n(\%))\end{array}$ \\
\hline Leptotene & 15.6 & $0(0.00)$ & $13(100.0)$ & 23.0 & 8 (8.4) & 87 (91.6) \\
\hline Zygotene & 33.3 & $25(69.4)$ & $11(30.6)$ & 21.4 & $63(70.0)$ & $27(30.0)$ \\
\hline Pachytene & 48.1 & $41(100.0)$ & $0(0.0)$ & 53.6 & 241 (100.0) & $0(0.0)$ \\
\hline Diplotene & 2.9 & $0(0.0)$ & $0(0.0)$ & 2.0 & $10(100.0)$ & $0(0.0)$ \\
\hline
\end{tabular}

pachytene (Fig. 6A1-A2), where ATR was seen to decorate almost the entire length of the SC (Fig. 6B). At late pachytene, ATR was predominantly located at the sex body and some ATR foci persisted on SCs, some of them marking telomeres (Fig. 6C). At the diplotene stage, a few ATR foci were present on the SCs.

\section{ATR distribution in human oocytes}

When ATR distribution in euploid oocytes was analyzed, it appeared that ATR distribution was similar to that in human spermatocytes described above. In euploid oocytes, ATR first appeared at leptotene as a few foci. At the zygotene stage, ATR was present at the unsynapsed and synapsed regions of the chromosomes. No remarkable differences in ATR staining were observed between 'late pairing' and 'synapsed' homologs at the late zygotene stage/early pachytene stage, mirroring the situation found in human spermatocytes. At early pachytene, ATR foci were present along the whole length of the SCs. At late pachytene, ATR foci remarkably decreased; some of them were located at the telomeres. Finally, oocytes at the diplotene stage had few ATR foci.

The same ATR distribution was found in trisomic 18 oocytes (Fig. 6D) and apparently no differential ATR staining was observed for the trivalent 18 at late zygotene/early pachytene.

\section{Discussion}

\section{Chromosomal dynamics in trisomic oocytes fit with dynamics described in euploid oocytes}

In this paper, a study of the dynamics of centromeres and telomeres, cohesin core and synapsis development in aneuploid female meiosis was performed.

According to previous observations made in human euploid oocytes (Prieto et al. 2004), development of REC8 chromosome cores and synapsis progression was not clearly found to be overtly altered in the presence of an extra copy of human chromosome 18, thus suggesting that cohesin core organization is not affected by the presence of an extra chromosome. The sequence of chromosomal events also mirrored those of euploid human oocytes (Roig et al. 2004). Therefore, it seems aneuploidy does not grossly affect chromosome core and SC formation, which agrees with previous reports (Luciani et al. 1976, Wallace \& Hulten 1983, Speed 1984, Cheng et al. 1995, 1998, Barlow et al. 2002).

The results of trivalent formation are similar to those described earlier for trisomic cases in human trisomy 21 oocytes (Wallace \& Hulten 1983, Speed 1984, Barlow et al. 2002) and in the rat (Martínez-Flores et al. 2001). However, thickening of the asynapsed chromosome core was not observed in preparations stained with REC8 and SYCP1, contrasting with previous observations made with electron microscopy (Wallace \& Hulten 1983, Speed 1984, Martínez-Flores et al. 2001). These results indicate that the different proteinaceous composition of the unpaired univalent is not related to meiotic cohesin REC8. IF data also corroborate the absence of SYCP3 and SYCP1 implication in this phenomenon, agreeing with previous observations (Barlow et al. 2002).

\section{An extra chromosome 18 does not alter pairing of other chromosomes}

Studies performed on Down's syndrome oocytes suggest that an extra chromosome may alter the pairing of other homologs (Cheng et al. 1998). When a comparison was made of the chromosome 13 pairing process found in Edwards' syndrome oocytes with control oocytes from a case at the same developmental stage, no obvious differences were found. Moreover, similar timing was presented. In contrast, Cheng et al. (1998) reported that chromosome 13 suffered a pairing delay in trisomy 21 oocytes. The differences in chromosome 13 pairing found between trisomy 21 and trisomy 18 oocytes may be caused by the frequent association existing between acrocentric NOR-bearing chromosomes 13 and 21, which is commonly observed both in euploid and aneuploid oocytes (I Roig and M Garcia, data not published).

Moreover, normal SYCP1 dynamics obtained in SYCP1 IF experiments also provide evidence that general chromosome synapsis progresses with no obvious differences compared with euploid oocytes (Roig et al. 2004).

Cheng et al. (1998) suggested that the possible interference caused by the extra chromosome 21 was diminished by the formation of a trivalent 21, mirroring observations made by other authors (Speed 1984, Rodriguez \& Burgoyne 2000). Thus, the high trivalent-18 formation efficiency observed in this study could explain the absence of an 

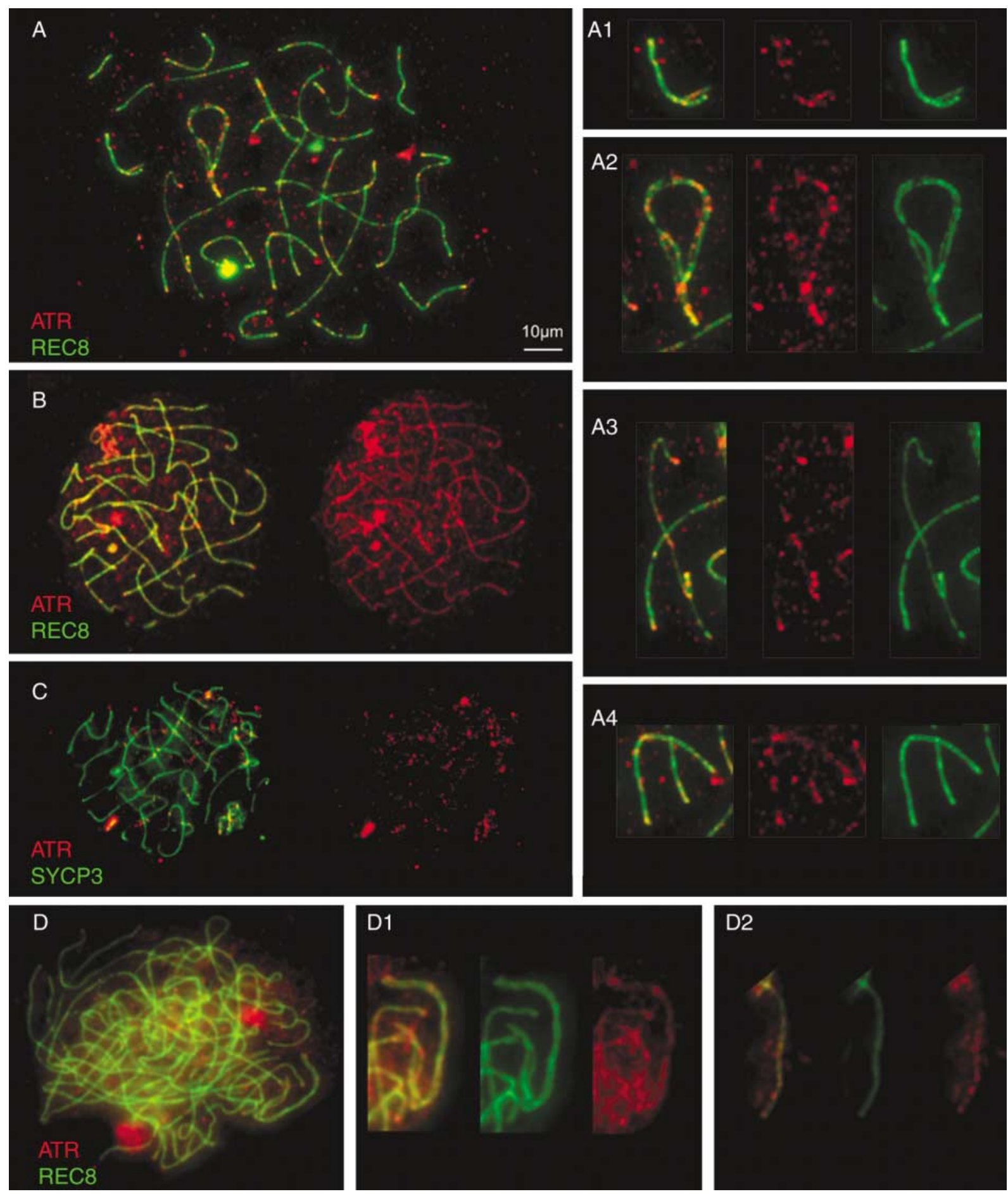

Figure 6 ATR distribution in human spermatocytes (A-C) and in a human trisomic 18 oocyte (D). (A) Late-zygotene spermatocyte in which almost all bivalents are paired. Some of the spermatocyte bivalents are enlarged (A1-A4) in order to show that ATR foci are present at the unpaired bivalents (A1 and A2), the sex body (A3) and the already-paired bivalents (A4); note that the ATR foci are present in both synapsed and asynapsed regions. (B) Early-pachytene spermatocyte having almost the whole length of the SCs covered with ATR; note the different brightness of the ATR foci and the telomeric position of some of them. (C) Late-pachytene spermatocyte showing that the number of ATR foci has decreased substantially, being at some telomeric and interstitial sites. ATR shows preferential staining for the sex body. (D) Late-zygotene oocyte mirroring the situation found in spermatocytes. A late-pairing bivalent (D1) presents a similar staining pattern to that of an already-paired bivalent (D2, only a fragment of the whole bivalent is zoomed). 
interfering effect of the extra chromosome in the other chromosome-pairing processes.

\section{Presence of an extra chromosome 18 delays bouquet resolution in trisomic oocytes}

The bouquet has been implied as a mediator of chromosome pairing (Loidl 1990, Scherthan 2001). A prolonged bouquet stage has been found between species with a similar genome size, but different chromosome complement (mouse vs cattle, where the latter has more chromosomes and thus a longer bouquet stage; see Pfeifer et al. 2001 for discussion) and disomic yeast strains (Rockmill \& Roeder 1998). The significant increase in bouquet frequency in trisomy 18 oocytes as compared with that of euploid cases of similar stage (Table 1) suggests that the oocyte modulates the bouquet stage in response to an extra chromosome.

Results obtained in methanol:acetic acid spreads suggest that chromosome- 18 pairing is an efficient process to achieve the trivalent condition. Trivalent formation is preceded in leptotene and zygotene by the formation of a bivalent plus a univalent, since the portion of oocytes with bivalent plus univalent was higher at leptotene and zygotene than in later stages (Table 2). Moreover, late-pairing trivalent 18s shown in Fig. 3 reflect the fact that normally a trivalent is formed from a bivalent plus a univalent. In all of the images, there seems to be an already-paired bivalent (black) to which the univalent (gray) tries to pair. This observation may reflect the requirement to form a trivalent due to the cell being allowed to pass a pachytenepairing checkpoint (Roeder \& Bailis 2000). It may be that the time required for bringing about the encounter of surplus chromosomes takes longer, and therefore bouquet topology lasts longer in aneuploid cells compared with euploid oocytes. It may also be that trivalent formation is a late event in prophase I and that the cells spend more time until pairing is finally achieved.

REC8-SYCP1 IF studies have revealed that the three chromosome 18 cores were not perfectly aligned in some early pachytene oocytes (Fig. 3), thus mirroring the findings of Barlow et al. (2002). Therefore, it could be that the bouquet also serves a role in mediating and/or maintaining homolog alignment. Alternatively, the presence of proteins involved in the recombination process, such as Replication Protein A (RPA) at the trivalent 18 which is formed at early pachytene (not shown) may reflect the fact that delayed recombination may have the potential to elicit a bouquet prolongation (Scherthan 2003, Roig et al. 2004).

Nevertheless, Edwards' syndrome is characterized by a general developmental delay, which could not be excluded as playing a role in the origin of the delayed bouquet resolution found in trisomic 18 oocytes. However, this seems unlikely since prophase sub-stage frequencies found in trisomy 18 (V70) and euploid (V54) cases were similar (Table 3 ). In the same way, preliminary results obtained in Down's syndrome oocytes (Robles P, Roig I, Garcia R, Egozcue J and Garcia M, unpublished data) seem to support the hypothesis that the extended bouquet could be due mainly to the presence of an extra chromosome.

\section{ATR in aneuploid oocytes}

The specific ATR decoration of the late-pairing bivalents found in mouse spermatocytes (Keegan et al. 1996, Moens et al. 1999, Baart et al. 2000) was not observed in human spermatocytes or oocytes in this study. Moreover, in the cells with late-pairing bivalents, ATR was most often located at the synapsed regions rather than at the asynapsed regions (Fig. 6A1 and A2). In this case, late zygotene spermatocytes showed that ATR was located mostly at the paired region of the $\mathrm{XY}$ bivalent (Fig. 6A3). This is in contrast to what has been reported for the male mouse where ATR was largely seen in asynapsed regions at the late zygotene stage and no ATR staining was observed at the pachytene stage except for the sex body (Keegan et al. 1996, Moens et al. 1999, Baart et al. 2000). A recent study performed on male mouse spermatocytes suggests a pachytene checkpoint function for ATR, which could be related to recombination rather than to synapsis (Perera et al. 2004). Our results seem to be in agreement with the latter hypothesis.

This is the first study in which a combination of FISH and IF techniques have been used in order to analyze homolog pairing in human trisomic oocytes. The usage of a dual approach enables the study of a large number of oocytes with accurate precision. Our results show that even in the presence of an extra chromosome the pairing process in human oocytes is generally completed, yielding evidence of the reliability of the homolog-chromosome pairing process in human female meiosis. However, further analysis in human euploid and aneuploid oocytes and their peculiarities in homolog pairing is required in order to gain deeper insights into the occurrence of this phenomenon in the human species.

\section{Acknowledgements}

We thank Dr I Prieto and Dr J L Barbero for antibody donation and comments and discussion about the manuscript. The authors also thank Dr F Vidal for her invaluable help. Finally, we would also like to thank Dr C Heyting for kindly donating SYCP3 and SYCP1 antibodies. I R is the recipient of a fellowship from the Ministerio de Educación, Cultura y Deporte (AP2000-0992), P R has a fellowship from the Ministerio de Sanidad, Grant FIS 02/0297 and R G has a fellowship from the Generalitat de Catalunya (2004FI 00953). This work was supported by a Ministerio de Sanidad grant, FIS 02/0297.

The English of this manuscript has been revised by a native English speaking instructor of English of this University. 
The authors declare that there is no conflict of interest that would prejudice the impartiality of this scientific work.

\section{References}

Baart EB, de Rooij DG, Keegan KS \& de Boer P 2000 Distribution of ATR protein in primary spermatocytes of a mouse chromosomal mutant: a comparison of preparation techniques. Chromosoma 109 139-142.

Barlow AL \& Hultén MA 1997 Sequential immunocytogenetics, molecular cytogenetics and transmission electron microscopy of microspread meiosis I oocytes from a human fetal carrier of an unbalanced translocation. Chromosoma 106 293-303.

Barlow AL, Tease C \& Hultén MA 2002 Meiotic chromosome pairing in fetal oocytes of trisomy 21 human females. Cytogenetics and Genome Research 96 45-51.

Bojko M 1983 Human meiosis VIII. Chromosome pairing and formation of the synaptonemal complex in oocytes. Carlsberg Research Communications 48 457-483.

Cheng EY, Chen YJ \& Gartler SM 1995 Chromosome painting analysis of early oogenesis in human trisomy 18 . Cytogenetics and Cell Genetics 70 205-210.

Cheng EY, Chen YJ, Bonnet G \& Gartler SM 1998 An analysis of meiotic pairing in trisomy 21 oocytes using fluorescent in situ hybridization. Cytogenetics and Cell Genetics 80 48-53.

Cohen PE \& Pollard JW 2001 Regulation of meiotic recombination and prophase I progression in mammals. Bioessays 23 996-1009.

Eijpe M, Heyting C, Gross B \& Jessberger R 2000 Association of mammalian SMC1 and SMC3 proteins with meiotic chromosomes and synaptonemal complexes. Journal of Cell Science 113 673-682.

Eijpe M, Offenberg H, Jessberger R, Revenkova E \& Heyting C 2003 Meiotic cohesin REC8 marks the axial elements of rat synaptonemal complexes before cohesins SCM1 $\beta$ and SMC3. Journal of Cell Biology 160 657-670.

Fawcett DW 1956 The fine structure of chromosomes in the meiotic prophase of vertebrate spermatocytes. Journal of Biophysical and Biochemical Cytology 2 403-406.

Garcia M, Dietrich M, Freixa L, Vink ACG, Ponsa M \& Egozcue J 1987 Development of the first meiotic prophase stages in human fetal oocytes observed by light microscopy. Human Genetics 77 $223-232$

Harper L, Golubovskaya I \& Cande W 2004 A bouquet of chromosomes. Journal of Cell Science 117 4025-4032.

Hassold T \& Hunt P 2001 To err (meiotically) is human: the genesis of human aneuploidy. Nature Reviews. Genetics 2 280-291.

Heyting C 1996 Synaptonemal complexes: structure and function. Current Opinion in Cell Biology 8 389-396.

Keegan KS, Holtzman DA, Plug AW, Christenson ER, Brainerd EE, Flaggs G, Bentley NJ, Taylor EM, Meyn MS, Moss SB et al. 1996 The Atr and Atm protein kinases associate with different sites along meiotically pairing chromosomes. Genes and Development 10 2423-2437.

Kim S, Kaminker P \& Campisi J 1999 TIN2, a new regulator of telomere length in human cells. Nature Genetics 23 405-412.

Lammers JH, Offenberg HH, van Aalderen M, Vink AC, Dietrich AJ \& Heyting C 1994 The gene encoding a major component of the lateral elements of synaptonemal complexes of the rat is related to X-linked lymphocyte-regulated genes. Molecular and Cellular Biology 14 1137-1146.

Lenzi ML, Smith J, Snowden T, Kim M, Fishel R, Poulos BK \& Cohen PE 2005 Extreme heterogeneity in the molecular events leading to the establishment of chiasmata during meiosis I in human oocytes. American Journal of Human Genetics 76 112-127.

Loidl J 1990 The initiation of meiotic chromosome pairing: the cytological view. Genome 33 759-778.
Luciani JM, Devictor M, Morazzani MR \& Stahl A 1976 Meiosis of trisomy 21 in the human pachytene oocyte. Chromosoma $\mathbf{5 7}$ 155-163.

Mahadevaiah SK, Turner JMA, Baudat F, Rogakou EP, de Boer P, Blanco-Rodríguez J, Jasin $\mathbf{M}$, Keeny S, Bonner W \& Burgoyne PS 2001 Recombinational DNA double-strand breaks in mice precede synapsis. Nature Genetics 27 271-276.

Martínez-Flores I, Egozcue J, Cabero L \& Garcia M 2001 Synaptic behaviour of some structural and numerical chromosome anomalies in female and male rats (Rattus norvegicus). Histology and Histopathology 16 701-706.

Martínez-Flores I, Egozcue J \& García M 2003 Synaptic process in rat (Rattus norvegicus): influence of the methodology on the results. Microscopy: Research Techniques 60 450-457.

Meuwissen RL, Offenberg HH, Dietrich AJ, Riesewijk A, van lersel M \& Heyting C 1992 A coiled-coil related protein specific for synapsed regions of meiotic prophase chromosomes. EMBO Journal 11 5091-5100.

Moens PB, Tarsounas M, Morita T, Habu T, Rottinghaus ST, Freire R, Jackson SP, Barlow C \& Wynshaw-Boris A 1999 The association of ATR protein with mouse meiotic chromosome cores. Chromosoma 108 95-102.

Moens PB, Kolas NK, Tarsounas M, Marcon E, Cohen PE \& Spyropoulos B 2001 The time course and chromosomal localization of recombination-related proteins at meiosis in the mouse are compatible with models that can resolve the early DNA-DNA interactions without reciprocal recombination. Journal of Cell Science 115 1611-1622.

Moses MJ 1968 Synaptonemal complex. Annual Review of Genetics $2363-412$

Offenberg HH, Schalk JAC, Meuwissen RLJ, van Aalderen M, Kester HA, Dietrich AJJ \& Heyting C 1998 SCP2: a major protein component of the axial elements of synaptonemal complexes of the rat. Nucleic Acids Research 26 2572-2579.

Pelttari J, Hoja MR, Yuan L, Liu JG, Brundell E, Moens P, Santucci-Darmanin S, Jessberger R, Barbero JL, Heyting $C$ et al. 2001 A meiotic chromosomal core consisting of cohesin complex proteins recruits DNA recombination proteins and promotes synapsis in the absence of an axial element in mammalian meiotic cells. Molecular and Cellular Biology 21 $5667-5677$.

Perera D, Perez-Hidalgo L, Moens PB, Reini K, Lakin N, Syvaoja JE, San-Segundo PA \& Freire R 2004 TopBP1 and ATR colocalization at meiotic chromosomes: role of TopBP1/Cut5 in the meiotic recombination checkpoint. Molecular Biology of the Cell 15 1568-1579.

Pezzi N, Prieto I, Kremer L, Perez Jurado LA, Valero C, Del Mazo J, Martinez AC \& Barbero JL 2000 STAG3, a novel gene encoding a protein involved in meiotic chromosome pairing and location of STAG3-related genes flanking the Williams-Beuren syndrome deletion. FASEB Journal 14 581-592.

Pfeifer C, Thomsen PD \& Scherthan H 2001 Centromere and telomere redistribution precedes homologue pairing and terminal synapsis initiation during prophase I of cattle spermatogenesis. Cytogenetics and Cell Genetics 93 304-314.

Pfeifer C, Scherthan H \& Thomsen PD 2003 Sex-specific telomere redistribution and synapsis initiation in cattle oogenesis. Developmental Biology 255 206-215.

Prieto I, Suja JA, Pezzi N, Kremer L, Martinez AC, Rufas JS \& Barbero JL 2001 Mammalian STAG3 is a cohesin specific to sister chromatid arms in meiosis I. Nature Cell Biology 3 761-766.

Prieto I, Pezzi N, Buesa JM, Kremer L, Barthelemy I, Carreiro C, Roncal F, Martinez A, Gomez L, Fernandez R et al. 2002 STAG2 and Rad21 mammalian mitotic cohesins are implicated in meiosis. EMBO Reports 3 543-550.

Prieto I, Tease C, Pezzi N, Buesa JM, Ortega S, Kremer L, Martínez A, Martínez-A C, Hultén MA, Barbero JL 2004 Cohesin component dynamics during meiotic prophase I in mammalian oocytes. Chromosome Research 12 197-213. 
Rockmill B \& Roeder GS 1998 Telomere-mediated chromosome pairing during meiosis in budding yeast. Genes and Development 12 $2574-2586$.

Rodriguez TA \& Burgoyne P 2000 Evidence that sex chromosome asynapsis, rather than excess $Y$ gene dosage, is responsible for the meiotic impairment of XYY mice. Cytogenetics and Genome Research 89 38-43.

Roeder GS \& Bailis JM 2000 The pachytene checkpoint. Trends in Genetics 16 395-403.

Roig I, Vanrell I, Ortega A, Cabero L, Egozcue J \& Garcia M 2003 The use of foetal ovarian stromal cell culture for cytogenetic diagnosis. Cytotechnology $\mathbf{4 1} 45-49$.

Roig I, Liebe B, Egozcue J, Cabero L, Garcia M \& Scherthan H 2004 Female-specific features of recombinational double-stranded DNA repair in relation to synapsis and telomere dynamics in human oocytes. Chromosoma $11322-33$.

Scherthan H 2001 A bouquet makes ends meet. Nature Reviews. Molecular Cell Biology 2 621-627.

Scherthan H 2003 Knockout mice provide novel insights into meiotic chromosome and telomere dynamics. Cytogenetics and Genome Research 103 235-244.

Scherthan H, Weich S, Schwegler H, Heyting C, Harle M \& Cremer T 1996 Centromere and telomere movements during early meiotic prophase of mouse and man are associated with the onset of chromosome pairing. Journal of Cell Biology 134 1109-1125.

Schmekel K \& Daneholt B 1995 The central region of the synaptonemal complex revealed in three dimensions. Trends in Cell Biology $5239-242$.

Solari AJ 1980 Synaptosomal complexes and associated structures in microspread human spermatocytes. Chromosoma 81 315-337.

Speed RM 1984 Meiotic configurations in female trisomy 21 foetuses. Human Genetics 66 176-180.

Wallace BMN \& Hulten MA 1983 Triple chromosome synapsis in oocytes from a human foetus with trisomy 21. Annals of Human Genetics 47 271-276.

Weier HU, Kleine HD \& Gray JW 1991 Labeling of the centromeric region on human chromosome 8 by in situ hybridization. Human Genetics 87 489-494.

Received 11 November 2004

First decision 29 November 2004

Revised manuscript received 14 January 2005

Accepted 24 January 2005 